\title{
Análisis del Comportamiento Presión-Temperatura en la Transición Isotrópico-Nemático del p-Azoxianisol al variar el alcance del Potencial
}

\author{
Eduardo García-Sánchez ${ }^{1 *}$, Francisco J. Martínez ${ }^{1}$ y Luís H. Mendoza-Huizar ${ }^{2}$ \\ (1) Universidad Autónoma de Zacatecas, Unidad Académica de Ingeniería Eléctrica, \\ Av. Ramón López Velarde, No. 801, 98060 Zacatecas, Zac.-México \\ (e-mail: eduardogarciasanchez@gmail.com; jamarux@hotmail.com) \\ (2) Universidad Autónoma del Estado de Hidalgo, Centro de Investigaciones Químicas, \\ Unidad Universitaria, Km. 4.5, Carretera Pachuca-Tulancingo, Pachuca, Hidalgo-México \\ (e-mail: hhuizar@uaeh.edu.mx)
}

\begin{abstract}
Resumen
En este trabajo se muestra un estudio del efecto del alcance del potencial en la teoría de perturbaciones de segundo orden desarrollada por García-Sánchez et al. para valores del alcance del potencial de $3.5,4,10,15,17$ y $20 \mathrm{con} a / b=3.5$. Se observó una mejor predicción teórica para un valor del alcance de potencial de 20 , lo que está de acuerdo con el planteamiento teórico en el desarrollo de la teoría de perturbación que incorpora la aproximación de largo alcance de Ponce y Renon. En esta aproximación el alcance de potencial se considera toma un valor de infinito. De este estudio es posible concluir que si durante la predicción teórica son considerados valores bastante grandes del alcance del potencial de pozo cuadrado en el modelo teórico, es posible tener mejores predicciones del comportamiento experimental presión-temperatura en la transición de fase isotrópico-nemático del cristal líquido P-azoxianisol.
\end{abstract}

\section{Analysis of Pressure-Temperature Behavior in the Isotropic-Nematic Transition of PAA to vary of SW Range}

\begin{abstract}
This work shows a study on the effect of the potential range in the Second Order Perturbation Theory developed by García-Sánchez et al. for potential range values of 3.5, 4, 10, 15, 17 and 20 with $a / b=3.5$, It was observed a better theoretical prediction for the potential range of 20 , which is in agreement with the theoretical formulation of the perturbation theory that includes the long range approximation of Ponce and Renon. In this approximation the potential range is considered to have an infinite value. From the present study, it is possible to conclude that if during the theoretical prediction, higher values of the square well potential range are considered in the theoretical model, it is possible to get a better prediction of the experimental behavior pressure-temperature in the isotropic-nematic phase transition for liquid crystal P-azoxianisol.
\end{abstract}

Keywords: phase transition, liquid crystal, p-Azoxianisol, isotropic-nematic, perturbation theory 


\section{INTRODUCCIÓN}

En su trabajo inicial Onsager (1949), demostró que a densidades suficientemente altas, un fluido que contiene moléculas con una geometría de varillas duras puede presentar en forma espontánea, una transición de fase Isotrópico-Nemático (I-N), es decir, una fase ordenada orientacionalmente, por lo cual, puede ser considerado como la piedra angular para teorías moleculares, cuyo objetivo sea describir dicha transición, dicha teoría presenta limitaciones al tratar de predecir cuantitativamente dicha transición para varillas duras de elongaciones intermedias, pero para varillas infinitamente grandes los resultados son exactos. Una de las alternativas que se presentan para mejorar las predicciones cuantitativas de dicha teoría ha sido tradicionalmente la inclusión de coeficientes viriales de orden superior (Vroege y Lekkerkerker, 1992). Aunque es importante resaltar que al efectuar dicha consideración, esto implica el manejo de una mayor cantidad de parámetros, lo cual se traduce de manera directa en un mayor costo computacional. Una de las aproximaciones más exitosas, ha sido el método de escalamiento propuesto por Lee (1988), en el cual, se propuso la utilización de la aproximación de Parsons (Parsons, 1979) para la función de distribución por pares para fluidos de coraza dura no-esférica. Esta aproximación ha sido re-examinada recientemente para esferocilindros (McGrother et al., 1996) y elipsoides de revolución rígidos (Camp et al., 1996).

Por otro lado, se han reportado en la literatura modelos que consideran la existencia de interacciones atractivas del tipo Maier-Saupe, los cuales permiten que dicha transición pueda ser descrita por medio de las interacciones atractivas de dispersión de largo alcance. En realidad, a pesar de la diferencia en los principios básicos, las teorías de Onsager y de Maier-Saupe son semejantes. En ambas teorías, la energía libre de Helmholtz se considera que esta compuesta por un término de entropía de mezclado, en el cual, las moléculas con orientaciones diferentes se consideran como especies diferentes, además, de un término de interacción energética. Lo anterior ha llevado al desarrollo de teorías en las que se consideran tanto las propiedades anisotrópicas del sistema como las interacciones atractivas del tipo Maier-Saupe (Tjipto-Margo y Evans, 1991; Williamson, 1998).

En la actualidad, hay una gran cantidad de estudios referentes a describir la transición de fase I-N del p-azoxianisol (PAA) utilizando diferentes modelos teóricos reportados en la literatura a 1 atmósfera. Dentro de dichos estudios, es importante resaltar el desarrollado por Williamson (1998), quien desarrolló una teoría de perturbaciones de primer orden tipo Barker-Henderson (Barker y Henderson, 1976), específicamente utilizando al modelo "Convex Peg" e incorporando la aproximación de largo alcance de Ponce y Renon (1976) en conjunción con la aproximación de desacoplamiento de Parsons (1979). Williamson (1998) efectuó la comparación entre la predicción teórica obtenida por él y algunos valores experimentales reportados en la literatura. El procedimiento de ajuste empleado en este trabajo fue el de Tjipto-Margo y Evans (1991) para dos corazas de elipsoides rígidos uniaxiales $3: 1$. En dicho procedimiento se fijó el valor del volumen molecular del PAA en $230 \AA^{3}$, el cual corresponde al de un esferoide, además de utilizar a $\varepsilon$ (profundidad del potencial de pozo cuadrado) como el parámetro de ajuste en conjunto con la temperatura de transición I-N. Los resultados obtenidos por Williamson, indican que el procedimiento de ajuste de Tjipto-Margo y Evans (1991) es poco eficaz, debido a que las predicciones teóricas cuantitativamente no se comparan favorablemente con los resultados experimentales, entre ellos el comportamiento Presión vs. Temperatura en la transición I-N. Sin embargo los resultados obtenidos por Williamson fueron mejores a los reportados previamente y en algunos casos existió una buena concordancia con los datos de simulación para corazas uniaxiales prolatas 3:1 y $\delta \geq 0$ (Samborski y Evans, 1993).

En este mismo contexto Martínez-Richa et al. (2003), utilizaron la teoría de perturbaciones de primer orden desarrollada por Williamson (1998) para analizar el mismo comportamiento experimental para el PAA. La diferencia principal con el trabajo original de Williamson (1998); fue el cálculo del volumen de la molécula PAA, el cual se calculó empleando los parámetros del eje principal del elipsoide $a$ y los semiejes del elipsoide de revolución $b$ y $c(b=c)$, previamente reportados por García-Sánchez et al. (2002). El volumen calculado, usando los parámetros a y $b$, permitió obtener un volumen molecular de $150 \AA^{3}$. Los resultados obtenidos por Martínez-Richa et al. (2003), para el caso de dos corazas de elipsoides rígidos uniaxiales 3.5:1, fueron mejores a los obtenidos por Williamson (1998), sin embargo no se obtuvo una predicción cuantitativa con el comportamiento experimental. 
Por otro lado, García-Sánchez et al. (2002) propusieron una teoría de perturbaciones a segundo orden en conjunción con la aproximación de Parsons (1979) y la aproximación de largo alcance de Ponce y Renon (1976) para analizar el comportamiento de transición de fase I-N en el cristal líquido PAA, pero a diferencia del trabajo de Williamson (1998), realizaron una optimización de geometría del PAA utilizando el método semiempirico PM3, esto con la finalidad de encontrar la estructura de mínima energía. Una vez obtenida esta estructura se determinó al elipsoide de revolución que envuelve a esta estructura para determinar el volumen de la molécula y a los valores a y $b$ para el caso uniaxial. Se encontró que $k=a / b \approx 3.5$ y que el volumen molecular era de $70 \AA^{3}$. Al comparar las predicciones teóricas para la razón 3.5:1 obtenidas por García-Sánchez et al. (2002) con las obtenidas por Williamson (1998) y Martínez-Richa et al. (2003) del comportamiento experimental de la molécula PAA; se encontró que los resultados de García-Sánchez et al. (2002) presentan la mejor predicción. Lo anterior sugiere que el valor del volumen molecular es esencial en la predicción del comportamiento presión contra temperatura y de la calidad de los resultados obtenidos.

Recientemente, García-Sánchez et al. (2007) analizaron el mismo comportamiento experimental utilizando la teoría de perturbaciones desarrollada por García-Sánchez et al. (2002) y propusieron calcular el volumen de la molécula aislada a través de la densidad de enlace $\left(\rho=0.08 \mathrm{e} / \AA^{3}\right)$ usando la teoría de funcionales de la densidad y el modelo de isodensidad del continuo polarizado (IPCM). En dicho estudio se encontró que el volumen de la molécula para el PAA era de $70.6 \AA^{3}$, Al efectuar el análisis para la razón 3.5:1 con los estudios descritos anteriormente, encontraron que este trabajo presentaba la mejor predicción teórica del comportamiento experimental. Adicionalmente, en este trabajo se encontró que los valores para a y b permiten considerar el valor de $k \approx 3,643$, por lo cual, en ese trabajo se efectuaron cálculos teóricos para las razones 3.6:1 y 3.7:1. Además, efectuaron un análisis del efecto del parámetro $k$ en la predicción teórica. Al comparar los resultados experimentales, para este caso se restringió el estudio para el caso particular en que $k=\lambda$, dicho estudio se efectúo para los casos reportados en la Literatura $(k=3$ y 3.5$)$ y los encontrados con el modelo IPCM $(k=3.6$ y 3.7). En dicho análisis se encontró que para el caso $k=\lambda=3$, la predicción teórica sobreestima el comportamiento experimental del PAA, pero en cambio, para el caso $k=\lambda=$ 3.5 se tiene la mejor predicción teórica del experimento.

Basándose en los trabajos anteriores, en el presente trabajo se propuso utilizar la teoría de perturbaciones desarrollada por García-Sánchez et al. (2002) y el modelo IPCM, pero a diferencia de los trabajos antes mencionados, se analizo el efecto que presenta el alcance del potencial de pozo cuadrado al considerar valores mayores que $k$ en el comportamiento experimental Presión vs. Temperatura del Cristal Líquido PAA. Esto en base a que hasta donde se sabe, no se ha analizado el considerar valores mayores de esta variable en los estudios teóricos que predicen el comportamiento de las propiedades experimentales del PAA. La razón de analizar el efecto que presenta el incrementar el valor del alcance del potencial de pozo cuadrado se basa en el hecho de que, al considerar valores mayores para el alcance, se incrementa la región que describe la contribución atractiva de largo alcance en la teoría, lo que sugiere que, teóricamente con esta consideración, se cumple satisfactoriamente la aproximación de largo alcance de Ponce y Renon (1976), misma que implícitamente presenta la consideración de que el alcance de potencial debe tender a infinito, es decir, dicha teoría debe ser más exacta, en la medida de que el alcance del potencial tome valores bastante grandes. En base a lo anterior se puede esperar que al utilizar valores bastante grandes para el alcance del potencial se tendrá una mejor predicción teórica del comportamiento experimental presión contra temperatura en la transición I-N del PAA, por lo que en este trabajo se propuso analizar dicha teoría para los casos en que valores del alcance del potencial de 3.5, 4, 10, $15,17 \mathrm{y}$ 20 con $k=3$. Es importante comentar que en el presente trabajo no consideraremos incorporar otro tipo de interacciones, como por ejemplo, contribuciones debido a la flexibilidad, campos externos, momentos dipolares y otros, ya que el objetivo del presente trabajo es mostrar que al utilizar teorías de perturbaciones que incorporen la aproximación de largo alcance de Ponce y Renon (1976) para predecir datos experimentales, estas requieren considerar valores grandes del alcance del potencial de pozo cuadrado con el objeto de obtener mejores predicciones. Al efectuar dicho análisis en el presente artículo, se encontró que el planteamiento teórico fue el correcto, ya que conforme el alcance de potencial del pozo cuadrado se incrementa, la predicción teórica del experimento es mejor. 


\section{MATERIALES Y MÉTODOS}

La teoría de perturbaciones a segundo orden desarrollada por García-Sánchez et al. (2002), se desarrolló en base al modelo Convex Peg (CP), el cual, considera una coraza dura convexa (contribución repulsiva) dentro de un potencial de pozo cuadrado (SW) esférico (contribución atractiva), la contribución repulsiva de corto alcance se considera que ocurre a través de la interacción entre corazas duras elipsoidales con longitudes del eje principal a y los semiejes ó ejes transversos $b$ y $c$, tomando la consideración de que para el caso uniaxial $b=c$, para la contribución atractiva se considera un potencial tipo pozo cuadrado con un alcance para el potencial $\lambda$ y una profundidad del potencial $\varepsilon$. Para predecir los diagramas de fase de los cristales líquidos, primero se evalúa la energía libre de Helmholtz para cada una de las fases I y N desarrollada por GarcíaSánchez et al. (2002), a continuación se efectúa la minimización de la energía libre utilizando el método de función de prueba propuesto por Onsager (1949) para cada una de las fases, con cada una de estas expresiones, se determina el valor de la presión y el potencial químico respectivamente. Finalmente, se aplican las condiciones de equilibrio, esto es, igualdad de temperaturas, presión y potencial químico para ambas fases. Los parámetros moleculares $a / b$ y el volumen de la molécula $V_{m}$, esenciales en la predicción teórica del comportamiento presión contra temperatura para el PAA, se consideraron que presentan valores de $a / b=3.5$ y $V_{m}=70.6 \AA^{3}$, obtenidos por García-Sánchez et al. (2002) y García-Sánchez et al. (2007), respectivamente.

\section{RESULTADOS}

En general, para los diferentes diagramas de fase utilizados en este trabajo, se encontró cualitativamente un solo tipo de diagrama de fase considerando valores de $a / b=3.5$ y diferentes alcances para el modelo de elipsoides rígidos uniaxiales. Este diagrama de fase, presentó una coexistencia Vapor-Líquido (V-L) con un punto crítico de temperatura $\left(T_{c}\right)$ a valores de densidad menores al punto triple. A valores de Temperatura $T>T_{c}$ en dicha coexistencia, existe una fase isotrópica homogénea. Además, existe un punto triple $\left(T_{t}\right)$ Vapor-Líquido-Nemático (V-L-N), a valores mayores de $T_{t}$ el diagrama presenta una región bifásica I-N a valores grandes de densidad. El análisis del comportamiento Presión vs. Temperatura, para la transición de fase I-N, involucró cálculos considerando $a / b=3.5$ y alcances de potencial para $\lambda=3.5,4,10,15,17$ y 20 que se presentan en las Tablas 1, 2, 3, 4, 5 y 6 respectivamente. En dichas tablas, $T^{\star}$ es la temperatura reducida y se expresa $T^{*}=k_{B} T / \varepsilon$, en donde $k_{B}$ es la constante de Boltzmann, T es la temperatura y $\varepsilon$ es la profundidad del potencial SW. Además en dichas tablas $\eta_{\text {iso }}$ y $\eta_{\text {nem }}$ son las fracciones de empaquetamiento isotrópico y nemático respectivamente, Por último, $P^{*}$ es la presión reducida expresada como $P^{*}=P V_{m} / k_{B} T$, donde $\mathrm{P}$ es la presión.

Tabla 1: Valores de $\mathrm{T}^{*}, \eta_{\text {iso }}, \eta_{\text {nem }} \mathrm{y}^{*}$ para el caso $\mathrm{a} / \mathrm{b}=3.5$ y $\lambda=3.5$

\begin{tabular}{|l|l|l|l|}
\hline$T^{*}$ & $\eta_{\text {iso }}$ & $\eta_{\text {nem }}$ & $P^{*}$ \\
\hline 2.8 & 0.50110 & 0.51858 & 5.205 \\
\hline 2.7 & 0.50040 & 0.51781 & 4.999 \\
\hline 2.5 & 0.49976 & 0.51793 & 4.605 \\
\hline 2.3 & 0.49885 & 0.51784 & 4.135 \\
\hline 2.1 & 0.49760 & 0.51753 & 3.572 \\
\hline 1.9 & 0.49616 & 0.51749 & 2.905 \\
\hline 1.7 & 0.49423 & 0.51750 & 2.088 \\
\hline 1.5 & 0.49159 & 0.51786 & 1.069 \\
\hline 1.4 & 0.48984 & 0.51827 & 0.458 \\
\hline 1.39 & 0.48966 & 0.51836 & 0.394 \\
\hline 1.38 & 0.48943 & 0.51837 & 0.326 \\
\hline 1.37 & 0.48922 & 0.51842 & 0.258 \\
\hline 1.36 & 0.48901 & 0.51850 & 0.191 \\
\hline 1.35 & 0.48879 & 0.51856 & 0.122 \\
\hline 1.34 & 0.48856 & 0.51863 & 0.052 \\
\hline 1.333 & 0.48840 & 0.51868 & 0.000 \\
\hline
\end{tabular}


Tabla 2: Valores de $\mathrm{T}^{*}, \eta_{\text {iso }}, \eta_{\text {nem }}$ y $\mathrm{P}^{*}$ para el caso $\mathrm{a} / \mathrm{b}=3.5$ y $\lambda=4$

\begin{tabular}{|l|l|l|l|}
\hline$T^{*}$ & $\eta_{\text {iso }}$ & $\eta_{\text {nem }}$ & $P^{*}$ \\
\hline 2.8 & 0.49955 & 0.52079 & 2.975 \\
\hline 2.6 & 0.49860 & 0.52102 & 2.434 \\
\hline 2.4 & 0.49744 & 0.52139 & 1.807 \\
\hline 2.2 & 0.49595 & 0.52196 & 1.070 \\
\hline 2.1 & 0.49595 & 0.52237 & 0.652 \\
\hline 2.0 & 0.49399 & 0.52289 & 0.193 \\
\hline 1.98 & 0.49376 & 0.52302 & 0.096 \\
\hline 1.97 & 0.49364 & 0.52308 & 0.047 \\
\hline 1.967 & 0.49360 & 0.52309 & 0.033 \\
\hline 1.964 & 0.49356 & 0.52312 & 0.017 \\
\hline 1.961 & 0.49353 & 0.52314 & 0.003 \\
\hline 1.960 & 0.49351 & 0.52314 & 0.000 \\
\hline
\end{tabular}

Tabla 3: Valores de $T^{*}, \eta_{\text {iso }}, \eta_{\text {nem }}$ y $P^{*}$ para el caso $a / b=3.5$ y $\lambda=10$

\begin{tabular}{|l|l|l|l|}
\hline$T^{*}$ & $\eta_{\text {iso }}$ & $\eta_{\text {nem }}$ & $P^{*}$ \\
\hline 40 & 0.50575 & 0.52774 & 2.641 \\
\hline 38 & 0.50543 & 0.52826 & 2.239 \\
\hline 36 & 0.50506 & 0.52886 & 1.792 \\
\hline 34 & 0.50462 & 0.52959 & 1.292 \\
\hline 32 & 0.50406 & 0.53047 & 0.729 \\
\hline 30 & 0.50337 & 0.53157 & 0.092 \\
\hline 29.8 & 0.50329 & 0.53169 & 0.023 \\
\hline 29.74 & 0.50327 & 0.53173 & 0.003 \\
\hline 29.732 & 0.50327 & 0.53173 & 0.000 \\
\hline
\end{tabular}

Tabla 4: Valores de $T^{*}, \eta_{\text {iso }}, \eta_{\text {nem }}$ y $\mathrm{P}^{*}$ para el caso $\mathrm{a} / \mathrm{b}=3.5$ y $\lambda=15$

\begin{tabular}{|l|l|l|l|}
\hline$T^{*}$ & $\eta_{\text {iso }}$ & $\eta_{\text {nem }}$ & $P^{*}$ \\
\hline 150 & 0.50660 & 0.52712 & 3.421 \\
\hline 140 & 0.50628 & 0.52771 & 2.936 \\
\hline 132 & 0.50597 & 0.52828 & 2.484 \\
\hline 120 & 0.50536 & 0.52936 & 1.702 \\
\hline 116 & 0.50512 & 0.52980 & 1.405 \\
\hline 112 & 0.50483 & 0.53029 & 1.087 \\
\hline 108 & 0.50451 & 0.53085 & 0.746 \\
\hline 104 & 0.50414 & 0.53147 & 0.377 \\
\hline 102 & 0.50394 & 0.53182 & 0.182 \\
\hline 101 & 0.50383 & 0.53199 & 0.082 \\
\hline 100.197 & 0.50374 & 0.53215 & 0.000 \\
\hline
\end{tabular}

Tabla 5: Valores de $T^{*}, \eta_{\text {iso }}, \eta_{\text {nem }}$ y $P^{*}$ para el caso $a / b=3.5$ y $\lambda=17$

\begin{tabular}{|l|l|l|l|}
\hline$T^{*}$ & $\eta_{\text {iso }}$ & $\eta_{\text {nem }}$ & $P^{*}$ \\
\hline 300 & 0.50766 & 0.52522 & 5.292 \\
\hline 290 & 0.50757 & 0.52538 & 5.120 \\
\hline 250 & 0.50715 & 0.52620 & 4.293 \\
\hline 200 & 0.50623 & 0.52793 & 2.793 \\
\hline 150 & 0.50411 & 0.53169 & 0.287 \\
\hline 146 & 0.50381 & 0.53218 & 0.012 \\
\hline 145.827 & 0.50379 & 0.53220 & 0.000 \\
\hline
\end{tabular}


Tabla 6: Valores de $T^{*}, \eta_{\text {iso }}, \eta_{\text {nem }}$ y $P^{*}$ para el caso $a / b=3.5$ y $\lambda=20$

\begin{tabular}{|l|l|l|l|}
\hline$T^{*}$ & $\eta_{\text {iso }}$ & $\eta_{\text {nem }}$ & $P^{*}$ \\
\hline 400 & 0.50712 & 0.52634 & 4.188 \\
\hline 350 & 0.50661 & 0.52732 & 3.316 \\
\hline 300 & 0.50581 & 0.52881 & 2.152 \\
\hline 250 & 0.50439 & 0.53133 & 0.519 \\
\hline 245 & 0.50419 & 0.53167 & 0.319 \\
\hline 240 & 0.50397 & 0.53205 & 0.111 \\
\hline 238 & 0.50388 & 0.53220 & 0.025 \\
\hline 237.6 & 0.50386 & 0.53224 & 0.008 \\
\hline 237.5 & 0.50385 & 0.53224 & 0.004 \\
\hline 237.42 & 0.50385 & 0.53225 & 0.000 \\
\hline
\end{tabular}

\section{DISCUSION}

Una de las limitaciones de la teoría desarrollada por García-Sánchez et al. (2002), seria la existencia de fases ordenadas en la posición, como por ejemplo un punto triple Vapor-Líquido-Esméctico (V-L$\mathrm{Sm}$ ) o Vapor-Líquido-Sólido Cristalino (V-L-K), esto puede ocurrir ya que el punto triple predicho por la teoría ocurre a una presión igual a cero, muy cerca a dichos puntos. Para el caso particular del Cristal Líquido PAA, este no presenta experimentalmente puntos triples V-L-Sm y V-L-K, por lo que el uso de dicho procedimiento es válido para estudiar la transición I-N del PAA.

Otro punto a considerar en la teoría desarrollada por García-Sánchez et al. (2002) radica en el hecho de que los valores del alcance del potencial son muy altos $(\lambda \geq 3.5)$, sin embargo, Williamson (1998) sugiere que una expansión de energía libre para la contribución SW del modelo Convex Peg que incorpore la aproximación de largo alcance de Ponce y Renon (1976) puede dar una buena predicción al satisfacer, el valor del alcance efectivo $\left(\lambda_{\text {eff }}\right)$ la condición de que $\lambda_{\text {eff }} \geq 2$. Lo anterior debido a que la aproximación de Parsons (1979), es un método en el cual, las propiedades termodinámicas de un fluido de coraza dura elipsoidal pueden ser mapeadas a otro fluido de esferas duras efectivo; donde, las esferas duras presentan el mismo volumen que las partículas no esféricas. Igualando los volúmenes del fluido del elipsoide con el del fluido esférico efectivo, el diámetro del fluido esférico efectivo $\left(\sigma_{\text {eff }}\right)$ en unidades de $\sigma_{0}$ puede ser escrito como

$\frac{\sigma_{e f f}}{\sigma_{0}}=\left(\frac{a b}{c^{2}}\right)^{1 / 3}$

Utilizando esta expresión, el alcance efectivo puede ser obtenido igualando los volúmenes del Convex Peg y el fluido efectivo, Por lo que se tiene que:

$\lambda_{\text {eff }}=\left(\frac{\lambda^{3}}{a b / c^{2}}\right)^{1 / 3}$

En virtud de lo anterior, en la Tabla 7 se muestra un concentrado de los alcances efectivos utilizados en este trabajo para predecir las propiedades experimentales del cristal líquido PAA. Como se puede observar en dicha tabla, todos los cálculos efectuado en este trabajo cumplen con la condición de que $\lambda_{\text {eff }} \geq 2$.

Es importante comentar, que para densidades altas, una teoría de perturbaciones a primer orden, como la que propuso Williamson (1998), presenta una buena descripción de los resultados teóricos, sin embargo, en este trabajo se opto por utilizar la teoría a segundo orden propuesta por GarcíaSánchez et al. (2002), esto con el objeto de mejorar las predicciones en los cálculos de los 
diagramas de fase a bajas densidades, esenciales en el estudio y predicción de la coexistencia V-L. Una limitación adicional, aparece por el hecho de utilizar una teoría de perturbaciones, descrita por una expansión a segundo orden en la energía libre de Helmholtz a altas temperaturas. Esto conlleva, a una deficiencia en la predicción teórica a bajas temperaturas. Sin embargo, Gil-Villegas et al. (1996), han demostrado que para fluidos esféricos SW caracterizados por una expansión a altas temperaturas en la aproximación de largo alcance, esta puede ser inadecuada cuando la temperatura es del orden de $0.6 T_{c}$, sin embargo, evaluando la temperatura en el punto triple I-L-N para los casos particulares presentados en este trabajo, los resultados mostrados en la Tabla 8 , muestran que es posible aplicar la teoría, ya que en general $T_{t}$ presenta valores entre $0.358 T_{c}$ y $0.381 T_{c}$

Tabla 7: Evaluación de la restricción para el alcance efectivo $\lambda_{\text {eff }} \geq 2$

\begin{tabular}{|c|c|c|}
\hline$a: b: c$ & $\lambda$ & $\lambda_{\text {eff }}$ \\
\hline $3.5: 1: 1$ & 3.5 & 2.305 \\
\hline & 4.0 & 2.634 \\
\hline & 10.0 & 6.586 \\
\hline & 15.0 & 9.879 \\
\hline & 17.0 & 11.196 \\
\hline & 20.0 & 13.172 \\
\hline
\end{tabular}

Tabla 8: Evaluación de la restricción $T_{t} / T_{c}$

\begin{tabular}{|c|c|c|}
\hline$\lambda$ & $T_{c}$ & $T_{t} / T_{c}$ \\
\hline 3.5 & 3.462 & 0.381 \\
\hline 4.0 & 5.212 & 0.376 \\
\hline 10.0 & 82.812 & 0.359 \\
\hline 15.0 & 279.714 & 0.358 \\
\hline 17.0 & 407.232 & 0.358 \\
\hline 20.0 & 663.171 & 0.358 \\
\hline
\end{tabular}

En lo que respecta al análisis del comportamiento presión-temperatura en la transición I-N para el PAA se procedió de la siguiente manera, primeramente, empleando el mismo procedimiento de ajuste realizado por Tjipto-Margo y Evans (1991), que consiste en emplear el valor experimental de la Temperatura del PAA en el punto triple de la transición I-N, que es de $408.9{ }^{\circ} \mathrm{K}$, dicho valor se sustituye en la expresión de $\mathrm{T}^{*}$ con el objetivo de determinar el valor de la profundidad del pozo cuadrado $\varepsilon$, teniendo este valor constante se determinan los demás valores de la temperatura para la transición con los otros valores de $\mathrm{T}^{*}$. Para determinar los valores de la presión se utilizo:

$P=P_{i}+\frac{\varepsilon T^{*} P^{*}}{V_{m}}$

En dicha expresión $P_{i}=1 \mathrm{~atm}=1.013$ bar, para los cálculos desarrollados en este trabajo se considero el valor del volumen molecular $V_{m}=70.6 \AA^{3}$ obtenido por García-Sánchez et al. (2007), con el cual, se pueden determinar los valores de la presión.

En la figura 1 se muestra la comparación de los resultados teóricos con los datos experimentales para el comportamiento Presión contra Temperatura en la transición de fase I-N del PAA. En ella, la línea recta representa a los datos experimentales obtenidos de Van Hecke (2001), las otras curvas representan las predicciones teóricas de dicho comportamiento para diferentes valores del alcance del potencial. Como se puede observar las predicciones están muy juntas, por lo que se analizaran únicamente dos curvas representativas que describan cualitativamente los resultados. La línea punteada representa la predicción teórica para el caso $\lambda=3.5$, que es el valor mas pequeño que se considero en este estudio, dicha predicción representa a la línea que tiene la pendiente mas pequeña 
y por lo tanto, la que esta mas alejada de los datos experimentales. Conforme se incrementa el valor del alcance del potencial, la pendiente de la curva teórica aumenta, lo cual da una mejor predicción del experimento, esto se puede ver cualitativamente si observamos a la línea con cuadros que representa el caso $\lambda=15$, esto en comparación con la línea punteada para el caso $\lambda=3.5$. En virtud de que es difícil observar que el comportamiento anterior lo cumplan las predicciones teóricas para los otros casos particulares con $\lambda=4,10,17$ y 20, ya que tendríamos a las curvas muy empalmadas y juntas, se selecciono un punto arbitrario de temperatura $\left(453.8^{\circ} \mathrm{k}\right)$ para determinar la presión, con el cual se pueda efectuar una comparación entre los diferentes casos.

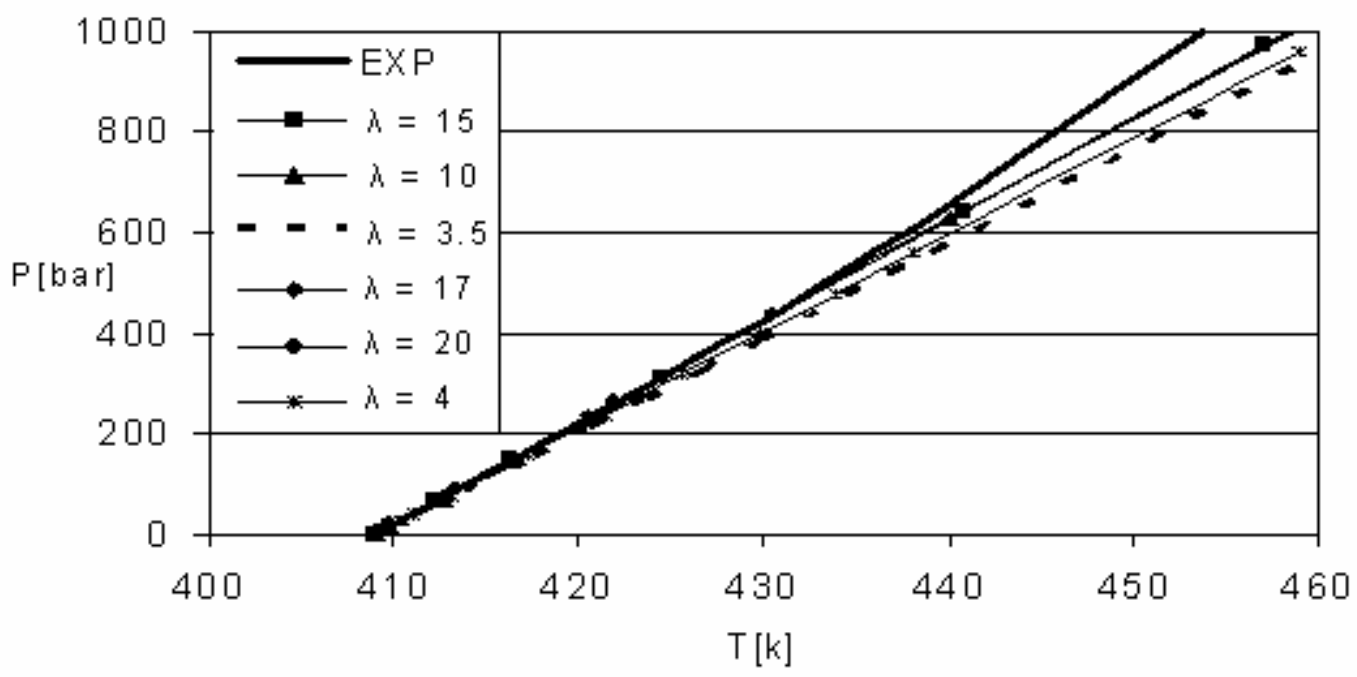

Fig. 1: Predicción teórica del comportamiento experimental del PAA (Van Hecke, 2001)

Tabla 9: Valor de $\mathrm{P}$ para diferentes valores de $\lambda$ a $453.8^{\circ} \mathrm{K}$

\begin{tabular}{|l|r|r|}
\hline & $\mathrm{T}\left[{ }^{\circ} \mathrm{k}\right]$ & $\mathrm{P}[\mathrm{bar}]$ \\
\hline Experimento & 453.8 & 1000.000 \\
\hline$\lambda=3.5$ & 453.8 & 835.980 \\
\hline$\lambda=4.0$ & 453.8 & 860.133 \\
\hline$\lambda=10.0$ & 453.8 & 903.042 \\
\hline$\lambda=15.0$ & 453.8 & 905.134 \\
\hline$\lambda=17.0$ & 453.8 & 905.408 \\
\hline$\lambda=20.0$ & 453.8 & 905.798 \\
\hline
\end{tabular}

Es posible notar a partir de los resultados mostrados en la Tabla 9, que conforme se incrementa el valor de $\lambda$ en la teoría de perturbaciones, se tiene una mejor predicción del experimento. Lo anterior es de esperarse ya que una de las consideraciones que se efectuaron para desarrollar dicha teoría, es que se supuso una interacción de largo alcance; por lo que los valores de $\lambda$ deben tender al infinito, o en su defecto, se deben de considerar valores bastante grandes. Dentro de los resultados mostrados en la Tabla 9 . Se pudiera esperar que si se consideran valores de $\lambda$ mayores, la predicción del experimento mejorara sustancialmente.

Adicionalmente, en este trabajo se presenta en la Tabla 10 un concentrado de otros parámetros esenciales en el estudio de las propiedades termodinámicas en la transición I-N para el PAA, en esta Tabla $\left\langle\mathrm{P}_{2}\right\rangle$ es el parámetro de orden, $\left\langle\mathrm{P}_{4}\right\rangle$ es el parámetro de orden cuadrupolar y $\Delta S / \mathrm{Nk}_{\mathrm{B}}$ describe la variación de la entropía adimensional. Entre los resultados que se pueden apreciar en esta Tabla, es el valor experimental de la Temperatura, dicho valor es exacto para algunas teorías, pero es importante comentar que esto se debe principalmente a que dicho valor es utilizado como un parámetro de ajuste. Para el caso del valor de la fracción de empaquetamiento en la fase nemática es predicho correctamente por Flapper y Vertogen (1981), utilizando una ecuación de estado para 
esferas duras, esto era de esperarse, ya que a diferencia de este trabajo, los demás trabajos que se muestran en la Tabla 10 utilizan teorías con elipsoides biaxiales por un lado y elipsoides uniaxiales por otro lado, los cuales sobreestiman la dependencia angular de las fuerzas atractivas, lo cual resulta en un corte de la transición de fase a bajas densidades. En lo que respecta a las predicciones teóricas para $\left\langle\mathrm{P}_{2}\right\rangle$ se puede observar que la mejor predicción teórica la efectúa Cotter (1977), esto es de esperarse, ya que dicho estudio analiza esferocilindros rígidos inmersos en un campo promedio anisotropico, además, por la misma situación, se puede esperar que este mismo trabajo, si se hubiera realizado, presentaría la mejor predicción para $\left\langle\mathrm{P}_{4}\right\rangle$, por último, para la predicción teórica de $\Delta S / N_{B}$, el trabajo que predijo adecuadamente fue Tjipto-Margo y Evans (1991), esto se debe a que en su trabajo utilizaron corazas biaxiales, lo que conlleva a predecir un orden orientacional reducido en la fase nemática.

Tabla 10: Comparación de varios parámetros obtenidos experimentalmente en la transición de fase I$\mathrm{N}$ a 1 atm de presión para el PAA con las predicciones teóricas reportadas en la Literatura.

\begin{tabular}{|c|c|c|c|c|c|c|c|c|}
\hline Trabajo & $a: b: c$ & $\lambda$ & $\mathrm{V}_{\mathrm{m}}\left[\AA^{3}\right]$ & $\mathrm{T}\left[{ }^{\circ} \mathrm{K}\right]$ & $\eta$ & $\left\langle\mathrm{P}_{2}\right\rangle$ & $\left\langle\mathrm{P}_{4}\right\rangle$ & $\Delta \mathrm{S} / \mathrm{Nk}_{\mathrm{B}}$ \\
\hline $\begin{array}{c}\text { Experimento } \\
\text { Van Hecke (2001) }\end{array}$ & & & & 408.9 & 0.620 & 0.550 & 0.180 & 0.170 \\
\hline Cotter (1977) & & & 230.0 & 410.4 & 0.445 & 0.542 & & 0.887 \\
\hline $\begin{array}{c}\text { Baron y Gelbart } \\
(1977)\end{array}$ & & & $\begin{array}{l}230.0 \\
295.0\end{array}$ & $\begin{array}{l}627.0 \\
208.0\end{array}$ & $\begin{array}{l}0.450 \\
0.550\end{array}$ & 0.930 & & $\begin{array}{l}3.500 \\
3.250\end{array}$ \\
\hline $\begin{array}{c}\text { Ypma y Vertogen } \\
(1978)\end{array}$ & & & & 409.0 & 0.596 & 0.473 & & 0.590 \\
\hline Flapper et al (1981) & & & $\begin{array}{l}230.0 \\
230.0\end{array}$ & $\begin{array}{l}409.0 \\
409.0\end{array}$ & $\begin{array}{l}0.620 \\
0.621\end{array}$ & $\begin{array}{l}0.437 \\
0.452\end{array}$ & & $\begin{array}{l}0.536 \\
0.567\end{array}$ \\
\hline Eldredge et al (1990) & & & $\begin{array}{l}230.0 \\
295.0\end{array}$ & $\begin{array}{l}434.0 \\
295.0\end{array}$ & $\begin{array}{l}0.510 \\
0.510\end{array}$ & $\begin{array}{l}0.970 \\
0.970 \\
\end{array}$ & & $\begin{array}{l}6.500 \\
6.500 \\
\end{array}$ \\
\hline $\begin{array}{c}\text { Tjipto-Margo y Evans } \\
(1991)\end{array}$ & $\begin{array}{l}3: 1.4: 1 \\
3: 1.45: 1\end{array}$ & 3.0 & $\begin{array}{l}230.0 \\
230.0\end{array}$ & $\begin{array}{l}406.0 \\
411.0\end{array}$ & $\begin{array}{l}0.523 \\
0.523\end{array}$ & $\begin{array}{l}0.400 \\
0.360\end{array}$ & $\begin{array}{l}0.110 \\
0.090\end{array}$ & $\begin{array}{l}0.230 \\
0.230\end{array}$ \\
\hline $\begin{array}{c}\text { Kromhout y Linder } \\
(1994)\end{array}$ & & & 230.0 & 408.0 & 0.380 & 0.200 & & \\
\hline $\begin{array}{l}\text { Linder y Kromhout } \\
\text { (1995) }\end{array}$ & & & 230.0 & 408.0 & 0.220 & & & 0.120 \\
\hline Willamson (1998) & $\begin{array}{l}3: 1: 1 \\
3: 1: 1\end{array}$ & $\begin{array}{l}3.0 \\
3.0\end{array}$ & $\begin{array}{l}100.0 \\
230.0\end{array}$ & $\begin{array}{l}408.8 \\
409.0 \\
\end{array}$ & $\begin{array}{l}0.548 \\
0.548 \\
\end{array}$ & $\begin{array}{l}0.570 \\
0.570 \\
\end{array}$ & $\begin{array}{l}0.230 \\
0.230 \\
\end{array}$ & $\begin{array}{l}0.670 \\
0.670 \\
\end{array}$ \\
\hline $\begin{array}{l}\text { García-Sánchez et al } \\
(2002)\end{array}$ & $\begin{array}{l}3: 1: 1 \\
3.5: 1: 1\end{array}$ & $\begin{array}{l}3.0 \\
3.5\end{array}$ & $\begin{array}{l}230.0 \\
70.0\end{array}$ & $\begin{array}{l}409.0 \\
409.0\end{array}$ & $\begin{array}{l}0.562 \\
0.519\end{array}$ & $\begin{array}{l}0.630 \\
0.690\end{array}$ & $\begin{array}{l}0.220 \\
0.300\end{array}$ & $\begin{array}{l}0.800 \\
1.070\end{array}$ \\
\hline $\begin{array}{c}\text { Martínez-Richa et al } \\
(2003)\end{array}$ & $3: 1: 1$ & 3.0 & 150.0 & 409.0 & 0.562 & 0.630 & 0.220 & 0.800 \\
\hline $\begin{array}{l}\text { García-Sánchez et al } \\
\text { (2007) }\end{array}$ & $\begin{array}{l}3.5: 1: 1 \\
3.6: 1: 1 \\
3.7: 1: 1\end{array}$ & $\begin{array}{l}3.5 \\
3.6 \\
3.7\end{array}$ & $\begin{array}{l}70.6 \\
70.6 \\
70.6 \\
\end{array}$ & $\begin{array}{l}408.9 \\
408.9 \\
408.9 \\
\end{array}$ & $\begin{array}{l}0.519 \\
0.511 \\
0.504 \\
\end{array}$ & $\begin{array}{l}0.690 \\
0.706 \\
0.741 \\
\end{array}$ & $\begin{array}{l}0.300 \\
0.319 \\
0.373 \\
\end{array}$ & $\begin{array}{l}1.070 \\
1.124 \\
1.340 \\
\end{array}$ \\
\hline Este trabajo & $\begin{array}{l}3.5: 1: 1 \\
3.5: 1: 1 \\
3.5: 1: 1 \\
3.5: 1: 1 \\
3.5: 1: 1 \\
3.5: 1: 1\end{array}$ & $\begin{array}{l}3.5 \\
4.0 \\
10 \\
15 \\
17 \\
20\end{array}$ & $\begin{array}{l}70.6 \\
70.6 \\
70.6 \\
70.6 \\
70.6 \\
70.6 \\
\end{array}$ & $\begin{array}{l}408.9 \\
408.9 \\
408.9 \\
408.9 \\
408.9 \\
408.9 \\
\end{array}$ & $\begin{array}{l}0.519 \\
0.523 \\
0.531 \\
0.532 \\
0.532 \\
0.532 \\
\end{array}$ & $\begin{array}{l}0.690 \\
0.695 \\
0.696 \\
0.696 \\
0.696 \\
0.696 \\
\end{array}$ & $\begin{array}{l}0.300 \\
0.304 \\
0.306 \\
0.306 \\
0.306 \\
0.306 \\
\end{array}$ & $\begin{array}{l}1.070 \\
1.061 \\
1.040 \\
1.039 \\
1.039 \\
1.039 \\
\end{array}$ \\
\hline
\end{tabular}

En virtud de los resultados anteriores se puede concluir que, si se desean predecir adecuadamente las propiedades termodinámicas de un cristal líquido en la transición I-N, se requiere de una teoría que incorpore interacciones debidas a campos externos y al medio en el que se encuentra, además de considerar en la teoría corazas biaxiales y otros parámetros adicionales que permitan incrementar la dependencia angular de las fuerzas atractivas. Por lo cual, podemos esperar por ejemplo, que utilizando una teoría basada en el modelo Gay-Berne se pueden obtener mejores predicciones, no solo para la transición I-N, sino también para analizar fases esmécticas (De Miguel et al., 2004) y 
discóticas (Bellier-Castella et al., 2004), esto debido a que dicho modelo presenta fases nemáticas biaxiales (Berardi y Zannoni, 2000) y por otro lado efectos de tipo dipolar (Fukunaga et al., 2004) y cuadrupolar (Whiters, 2003) esenciales para tener un modelo que permita predecir una gama más amplia de propiedades termodinámicas con mejores resultados.

\section{CONCLUSIONES}

Analizando los resultados mostrados anteriormente en la sección de resultados y discusión, se encontró que al emplear la teoría de perturbaciones a segundo orden desarrollada por GarcíaSánchez et al. (2002), en conjunción con cálculos efectuados para determinar el valor del volumen molecular y de k para el PAA, se obtienen mejores predicciones teóricas para el comportamiento presión contra temperatura en la transición I-N, si se consideran valores grandes para el alcance del potencial de pozo cuadrado del modelo teórico y no para el caso tradicional, en el que se consideraba que $\lambda \mathrm{y} \mathrm{k}$ eran iguales. Es muy importante resaltar lo anterior, ya que si se desean obtener mejores predicciones teóricas del comportamiento experimental, al utilizar una teoría de perturbaciones que incorpore la aproximación de largo alcance de Ponce y Renon (1976), no únicamente para el caso del PAA, sino para cualquier otro Cristal Líquido, se debe considerar un valor bastante grande del alcance del potencial. Lo anterior nos permite concluir que a mayor valor del alcance del potencial, mejor predicción del comportamiento presión contra temperatura en la transición I-N se tendrá. Es importante comentar adicionalmente, que en este trabajo no se considero necesario incorporar una contribución debido a la flexibilidad, ya que la molécula PAA presenta una estructura rígida, aunque para otros casos esta contribución puede ser fundamental en la calidad de los resultados que se obtengan, como por ejemplo la molécula del HOAOB (García-Sánchez et al., 2007).

\section{AGRADECIMIENTOS}

Este trabajo fue financiado por PROMEP/103.5/04/1420, UAZ-2007-35691) y el Consejo Zacatecano de Ciencia y Tecnología (COZCYT)

\section{REFERENCIAS}

Barker, J.A. y D. Henderson; What is "liquid"? Understanding the states of matter, Reviews of Modern Physics: 48 (4), 587-671 (1976).

Baron, B.A. y W.M. Gelbart; Molecular shape and volume effects on the orientational ordering of simple liquid crystals, Journal Chemical Physics: 67 (12), 5795-5801 (1977)

Bellier-Castella, D., D. Caprion y J. P. Ryckaert; Surface ordering of diskotic liquid crystals, Journal Chemical Physics: 121 (10), 4874-4883 (2004)

Berardi, R. y C. Zannoni; Do thermotropic biaxial exist? A Monte Carlo study of biaxial Gay-Berne particles, Journal Chemical Physics: 113 (14), 5971-5979 (2000)

Camp, P.J y otros cuatro autores; The isotropic-nematic phase transition in uniaxial hard ellipsoid fluids: Coexistence data and the approach to the Onsager limit, Journal Chemical Physics: 105(7), 2837-2849 (1996)

Cotter, M.A.; Hard spherocylinders in an anisotropic mean field: A simple model for a nematic liquid crystal, Journal Chemical Physics: 66 (3), 1098-1106 (1977)

De Miguel, E., E. Martín del Río y F. J. Blas; Stability of smectic phases in the Gay-Berne model, Journal Chemical Physics: 121 (22), 11183-11194 (2004)

Eldredge, C. ., H.T. Heath, B. Linder y R.A. Kromhout; Use of dispersion potential anisotropy in the theory of nematic liquid crystals. I. The mean-field potential, Journal Chemical Physics: 92 (7), 6225$6234(1990)$ 
Flapper, S.D.P. y G. Vertogen; The equation of state for nematics revisited, Journal Chemical Physics: 75 (7), 3599-3607 (1981)

Fukunaga, H., J. Takimoto y M. Doi; Molecular dynamics simulation study on the phase behavior of the Gay-Berne model with a Terminal dipole and a flexible tail, Journal Chemical Physics: 120 (16), 7792-7800 (2004)

García-Sánchez y otros cuatro autores; Análisis del comportamiento presión-temperatura y otras propiedades termodinámicas para los cristales líquidos PAA, 5CB y HOAOB utilizando el modelo convex peg y la teoría de los funcionales de la densidad en la transición isotrópica-nemática, Revista Mexicana de Física: 53 (3), 179-188 (2007)

García-Sánchez, E. y otros cuatro autores; Predicting the Phase Diagram of a Liquid Crystal Using the Convex Peg Model and the Semiempirical PM3 Method, Journal of Chemical Physics A: 106 (43), 10342-10349 (2002)

Gil-Villegas, A., F. Del Río y A.L. Benavides; Deviations from corresponding-states behavior in the vapor-liquid equilibrium of the square-well fluid, Fluid Phase Equilibria: 119 (1-2), 97-112 (1996)

Kromhout, R.A. y B. Linder; Application of the total-correlation function formulation of ordered system to nematic hard ellipsoids, Journal Chemical Physics: 101 (2), 1755-1756 (1994)

Lee, S.; The Onsager-type theory ordering of finite-length hard ellipsoid, Journal Chemical Physics: 89 (11), 7036-7037 (1988)

Linder, B. y R.A. Kromhout; Nematic-isotropic phase transition: Hard spheroids with London interaction, Journal Chemical Physics: 102 (16), 6566-6569 (1995)

Martínez-Richa, A., E. García-Sánchez y D.C. Williamson; El Convex Peg aplicado al p-azoxianisol, Revista Mexicana de Ingeniería Química: 2 (1), 35-41 (2003)

McGrother, S.C., D.C. Williamson y G. Jackson; A re-examination of the phase diagram of hard spherocylinders, Journal Chemical Physics: 104 (17), 6755-6771 (1996)

Onsager, L.; The effects of shape on the interaction of colloidal particles, Annals of the New York Academy of Sciences: 51 (4), 627-659 (1949)

Parsons, D.; Nematic ordering in a system of rods, Physical Review A: 19 (3), 1225-1230 (1979)

Ponce, L. y H. Renon; Analytical equation for the Helmholtz free energy of a pure fluid, using the perturbation theory and a square well potential, Journal Chemical Physics: 64 (2), 638-640 (1976)

Samborski, A. y G.T. Evans; A phase diagram for non-spherical molecules with spherical square wells Isotropic and nematic phases, Molecular Physics: 80 (5), 1257-1261 (1993).

Tjipto-Margo, B. y G. T. Evans; A van der Waals theory of nematic liquid crystals: a convex peg in a round hole potential, Molecular Physics: 74 (1), 85-101 (1991)

Van Hecke, G.R.; "Phase transitions and the effects of pressure, Thermodynamics", Physical Properties of Liquid Crystals: Nematics, EMIS Datareviews Series; No. 25, Published by: INSPEC, The Institution of Electrical Engineers, pp 127-140, London, United Kingdom (2001)

Vroege, G.J. y H.N.W. Lekkerkerker; Phase transitions in lyotropic colloidal and polymer liquid crystal, Report on Progress Physics: 55 (8), 1241-1328 (1992)

Williamson, D.C.; the "Convex Peg" model: the long range approximation, Molecular Physics: 95 (2), 319-329 (1998) 
Withers, I. M.; Effects of longitudinal quadrupoles on the phase behavior of a Gay-Berne fluid, Journal Chemical Physics: 119 (19), 10209-10223 (2003)

Ypma, J.G. J. y G. Vertogen; Equation of state for nematic liquid Crystals, Physics Review A: 17 (4), $1490-1503(1978)$ 